\title{
Coupling Device with Resonant Cavities Based on Periodic Dielectric Waveguides
}

\author{
Ruei-Chang Lu, Yu-Lung Jang \\ Department of Electronic Engineering, National I-Lan University, Chinese Taipei \\ Email: revolution-42@hotmail.com
}

Received 2013

\begin{abstract}
In this paper, we propose a coupling device based on periodic dielectric waveguide. In order to reduce the device size, two resonant cavities are added so that the light can be coupled between two periodic dielectric waveguides. Plane wave expansion method and two dimension finite difference time domain methods are used to analyze this device. The coupled transmission ratio of this device at the wavelength of $1550 \mathrm{~nm}$ is above $94 \%$.
\end{abstract}

Keywords: Periodic Dielectric Waveguides; Direction Coupler

\section{Introduction}

Periodic dielectric waveguides (PDWGs) have been studied much in recent years. The structure of PDWG is similar to that of photonic crystal waveguide (PCWG). PDWG can be formed by dielectric cylinder array in air or air-hole array in a dielectric [1,2]. They are dielectric structures with lattice periodicity in one, two or three dimensions. Though the guiding methods are not the same, PDWG and PCWG can form similar device in optics communication, such as, multimode-interference (MMI)[3], optical power splitter based on T-junction [4] or Y-junction, polarization device [5], channel drop filter [6], direction coupler, etc. These device based on PDWG have the advantages of the structure compactness and flexibility. In order to reduce device size, we design a coupling device with two resonant cavities. The resonant cavity is a kind of point defects. By introducing point and/or line defects in direction coupler, the light from the PDWG can be guided to the other one.

\section{Design}

The coupling device we designed has two resonant cavities to couple the propagating light between two PDWGs, as show in Figure 1. In our design, the simulated parametric of dielectric constant is $\varepsilon=11.56$ (i.e. the index of refraction is $n=3.4$ ), the radius of the cylinder is set $r$ $=0.45 * a$, and the radius of cavity is $R=1.70 * a$, where $a$ is the center to center distance between two adjacent cylinders. We set the distance of the two PDWGs $4.5 a$, because the distance between these two PDWGs should be far enough not to couple each other. The distance of the two central cavities is $2 * \mathrm{r}_{\mathrm{d}}$, and $\mathrm{r}_{\mathrm{d}}=1.75 * a$. When the light is guided form port 1 to port 2, we define the transmission power ratio $\eta_{2}$. Similarly, when the light is guided from port 1 to port 3 , the transmission ratio is $\eta_{3}$.

\section{Simulation and Results}

The band of TE mode was calculated by plane wave expansion method (PWE). Figure 2 shows the band structure for the $a \times 14.5 a$ supercell by the dash frame. The shaded region represents extended mode region. In the band structure, we use the frequency from 0.10 to 0.16 . In this frequency range, two modes are very closed. That means the light can not coupled to the other one. Unless the device is long enough. However, using FDTD simulation result, it shows the transmission ratio of different ports versus normalized frequency $a / \lambda$ in Figure 3. We can see that most power guided to port 3 at $a / \lambda=0.145$ and port 2 at $a / \lambda=0.155$. Figure 4 shows the corresponding propagating field of these two cases. With $a=$ $225 \mathrm{~nm}$, the input wavelength of Figure 4(a) is $1550 \mathrm{~nm}$,

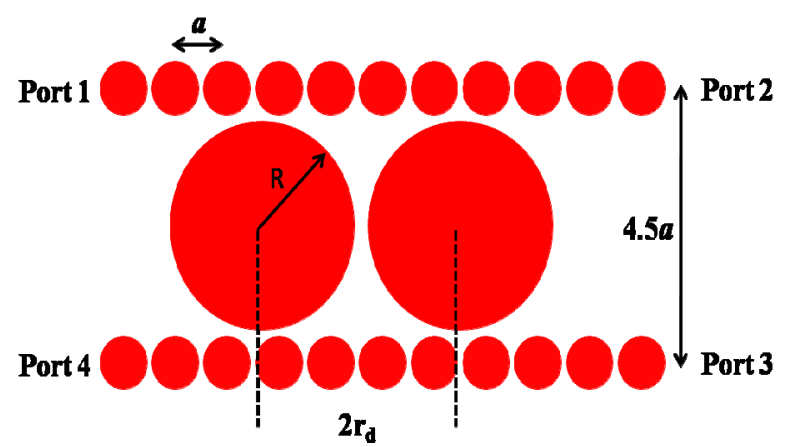

Figure 1. Schematic diagram of the device. 


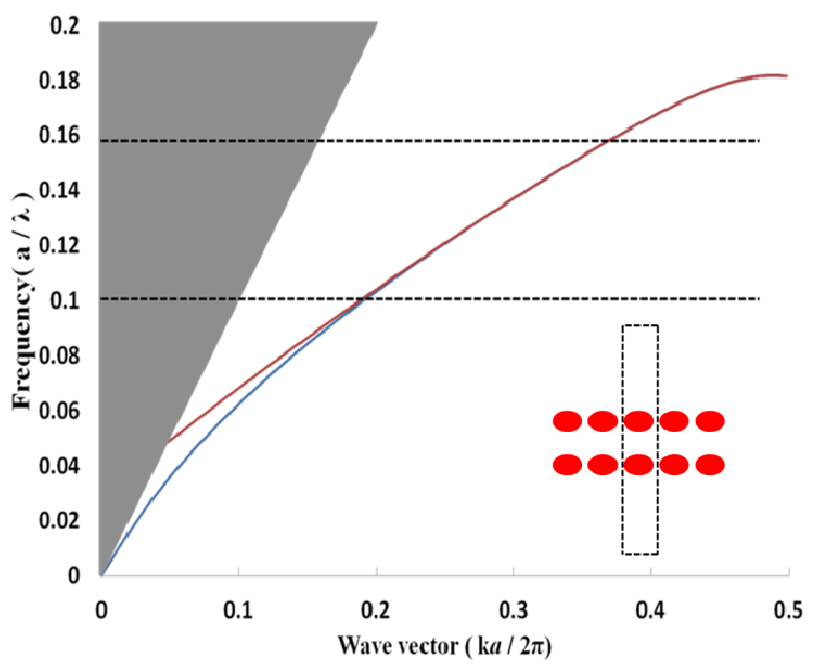

Figure 2. A diagram of two rows PDWGs. The dash frame shows the supercell for PWE method. Between the two dashes line is our operating frequency from 0.10 to 0.16 . The shaded region represents extended mode region.

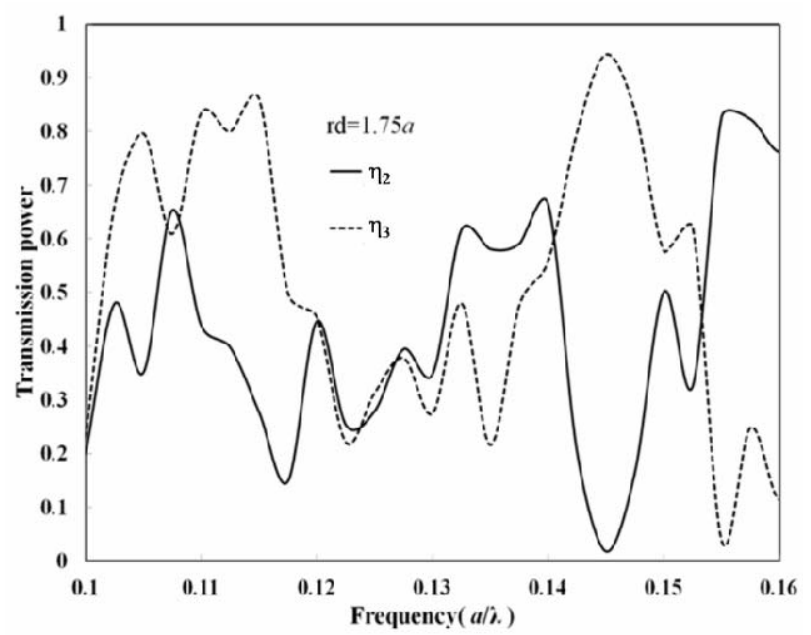

Figure 3. The relationship of transmission ratio and frequency.

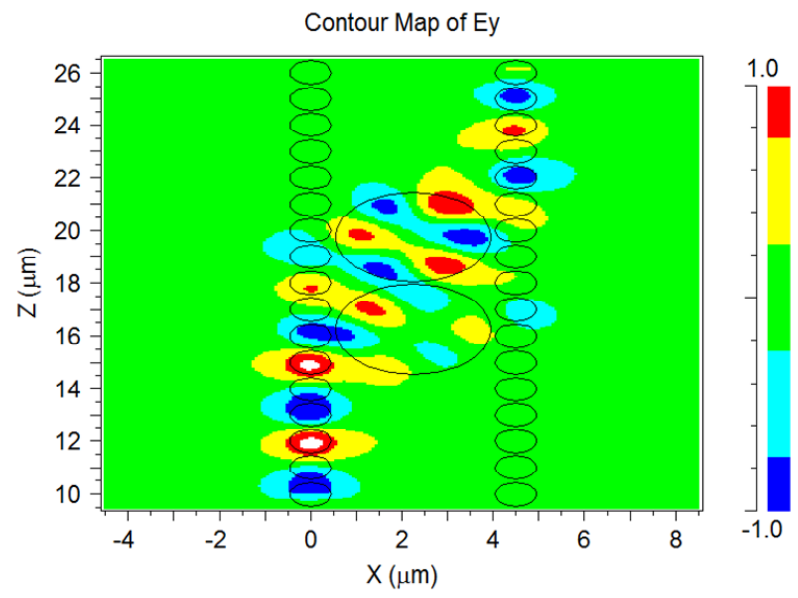

(a)

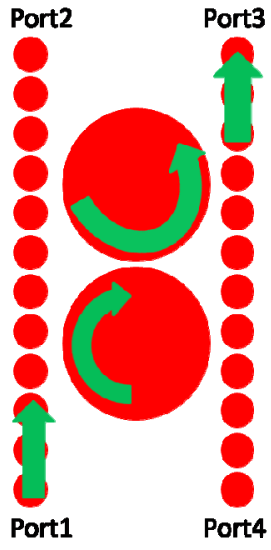

(b)

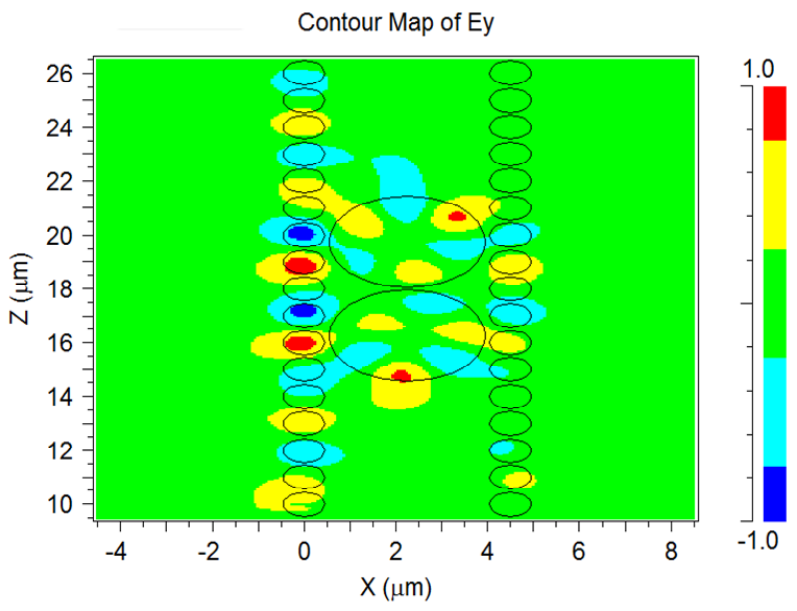

(c)

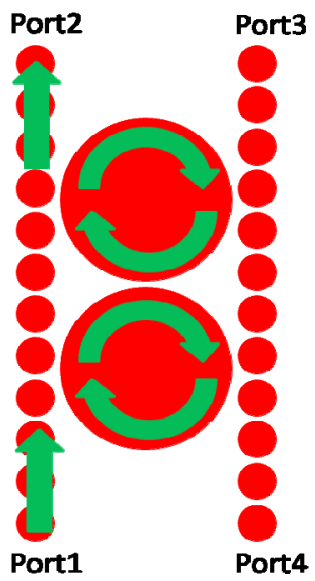

(d)

Figure 4. (a) Propagation field for input wavelength 1550 nm; (b) The light round the two resonant cavities with different directions; (c) Propagation field for input wavelength $1450 \mathrm{~nm}$; (d) The light round the two resonant cavities with the same direction.

and we can see that the light is guided mainly into port 3 with normalized transmission power $94 \%$. The input 
wavelength of Figure 4(c) is $1450 \mathrm{~nm}$, and we can see that the light is guided mainly into port 2 with lower transmission power $82 \%$.

We explain this wave how to propagate from port 1 to port 3 in Figure 4(b). The light surrounded to the two resonant cavities, upper cavity and lower cavity. The upper cavity is counter clockwise and the lower cavity is clockwise. There is a $180^{\circ}$ phase difference between the two resonant cavities. Due to the electric field with positive and negative caused to offset by the amplitude. So the light can not travel to port 4.

Another case is shown in Figure 4(d), there are clockwise in both resonant cavities. The above cavity couple to right PDWG but the light propagates several nanometers to couple to below cavity immediately. The same condition occur to the below cavity that port 4 gets about $5 \%$ loss come from the below cavity.

\section{Conclusions}

In order to reduce device size, we have proposed a coupling device with two cavities in PDWG. PDWG just likes a grating pattern, as $a=225 \mathrm{~nm}$, we can guide the light to different ports at $1550 \mathrm{~nm}$ and $1450 \mathrm{~nm}$ using plane wave expansion method and finite-difference time-domain method. The proposed device can be used in the future optical communication systems.

\section{REFERENCES}

[1] W. Zhang, J. Liu, W. Huang and W. Zhao, "Ultracom-pac t Wave Plates by Air Holes Periodic Dielectric Wave-Guides” SPIE Photonic and Phononic Crystal Materials and Devices X, 2010.

[2] K.-Y. Lee, C.-H. Lee, C.-H.Tsai, M.-H. Jiang, J.-F. Shueh, L. J. Han, Y.-L. Jeng and Y.-S. Chao, "FDTD Analysis of Point-Defected Periodical Dielectric Waveguide,” Optical and Quantum Electronics, 2009, pp. 91-98.

[3] S. Q. Zeng, Y. Zhang, B. J. Li and E. Yue-Bun Pun, "Ultrasmall Optical Logic Gates Based on Silicon Periodic Dielectric Waveguides” Fundamentals and Application, 2010, pp. 32-37.

[4] H. T. Guo, Y. Zhang and B. J. Li, "Periodic Dielectric Waveguide-Based Cross- and T-Connections with A Resonant Cavity at the Junctions,” Optics Communications, 2011, pp. 2292-2297.

[5] Y. Morita, Y. Tsuji and K. Hirayama, "Proposal for A Compact Resonant-Coupling-Type Polarization Splitter Based on Photonic Crystal Waveguide With Absolute Photonic Bandgap,” IEEE Photonics Technology. Letters, 2008, pp. 93-95.

[6] L. Li and G. Q. Liu, "Multi-Channel Drop filters Using Photonic Crystal Ring Resonators,” Optik-International Journal for Light and Electron Optics, 2012, pp. 167-170. 